\title{
Physician's Global Assessment in Psoriatic Arthritis: A Multicenter GRAPPA Study
}

\author{
Alberto Cauli, Dafna D. Gladman, Alessandro Mathieu, Ignazio Olivieri, Giovanni Porru, \\ Paul P. Tak, Claudia Sardu, Raffaele Scarpa, Antonio Marchesoni, William J. Taylor, \\ Carlo Salvarani, Joachim Kalden, Ennio Lubrano (i), Sueli Carneiro, Matteo Piga (1), \\ Alberto Floris, Francesca Desiati, John A. Flynn, Salvatore D’Angelo, Arno W.R. van Kuijk, \\ Maria Grazia Catanoso, Francesco Caso, Paolo Contu, Ilona Ujfalussy, Philip S. Helliwell, \\ and Philip J. Mease, for the GRAPPA 3PPsA Study Group
}

ABSTRACT. Objective. Physician's global assessment (PGA) of disease activity is a major determinant of therapeutic decision making. This study assesses the reliability of the PGA, measured by means of $0-100 \mathrm{~mm}$ visual analog scale (VAS), and the additional use of separate VAS scales for musculoskeletal (PhysMSK) and dermatologic (PhysSk) manifestations in patients with psoriatic arthritis (PsA).

Methods. Sixteen centers from 8 countries enrolled 319 consecutive patients with PsA. PGA, PhysMSK, and PhysSk evaluation forms were administered at enrollment (W0) and after 1 week (W1). Detailed clinical data regarding musculoskeletal (MSK) manifestations, as well as dermatological assessment, were recorded.

Results. Comparison of W0 and W1 scores showed no significant variation (intraclass correlation coefficients were PGA 0.87, PhysMSK 0.86, PhysSk 0.78), demonstrating the reliability of the instrument. PGA scores were dependent on PhysMSK and PhysSk ( $p<0.0001)$ with a major effect of the MSK component $(\mathrm{B}=0.69)$ compared to skin $(\mathrm{B}=0.32)$. PhysMSK was correlated with the number of swollen joints, tender joints, and presence of dactylitis $(\mathrm{p}<0.0001)$. PhysSk scores were correlated with the extent of skin psoriasis and by face, buttocks or intergluteal, and feet involvement $(\mathrm{p}<0.0001)$. Finally, physician and patient assessments were compared showing frequent mismatch and a scattered dot plot: PGA versus patient's global assessment $(r=0.36)$, PhysMSK versus patient MSK $(r=0.39)$, and PhysSk versus patient skin $(r=0.49)$.

Conclusion. PGA assessed by means of VAS is a reliable tool to assess MSK and dermatological disease activity. PGA may diverge from patient self-evaluation. Because MSK and skin/nail disease activity may diverge, it is suggested that both PhysMSK and PhysSk are assessed. (First Release June 15 2018; J Rheumatol 2018; 45:1256-62; doi:10.3899/jrheum.171183)

Key Indexing Terms:

PSORIATIC ARTHRITIS

INSTRUMENTS

ASSESSMENT MEASURES DOMAINS

PHYSICIAN'S GLOBAL ASSESSMENT

From the Department of Medical Sciences, Policlinico of the University of Cagliari; Rheumatology Unit, and Department of Public Health, University of Cagliari, Cagliari; Rheumatology Department of Lucania, San Carlo Hospital of Potenza and Madonna delle Grazie Hospital of Matera, Matera; Rheumatology Research Unit, Department of Clinical and Experimental Medicine, University "Federico II," Naples; Department of Rheumatology, ASST Gaetano Pini-Centro Traumatologico Ortopedico (CTO), Milan; Rheumatology Unit, Azienda USL-IRCCS di Reggio Emilia and Università di Modena e Reggio Emilia, Modena and Reggio Emilia; Dipartimento di Medicina e Scienze della Salute, Università degli Studi del Molise, Campobasso, Italy; University of Toronto, Toronto Western Hospital, Toronto, Ontario, Canada; Academic Medical Center/University of Amsterdam; Amsterdam Rheumatology and Immunology Center Reade, Amsterdam, the Netherlands; Rehabilitation Teaching and Research Unit, Department of Medicine, Wellington School of Medicine and Health Sciences, University of Otago, Wellington, New Zealand; Medizinische Klinik III mit Poliklinik, Friedrich-AlexanderUniversität Erlangen-Nuernberg, Erlangen, Germany; Sector of Dermatology, HUCFF School of Medicine, Federal University of Rio de Janeiro and Sector of Dermatology, HUPE School of Medical Sciences, State University of Rio de Janeiro, Rio de Janeiro, Brazil;

Spondyloarthritis Program, Johns Hopkins University, School of Medicine, Baltimore, Maryland; Swedish Medical Centre and University of Washington School of Medicine, Seattle, Washington, USA; Department of Rheumatology, Semmelweis University, Budapest, Hungary; Leeds Institute of Molecular Medicine, University of Leeds, Leeds, UK.
Funded by Regione Autonoma della Sardegna - L.R. n. 7/2007 annualità 2012, CRP-60098, linea A2, "Consorzio internazionale PEAC: meccanismi patogenetici nelle Spondiloartriti."

A. Cauli, MD, PhD, Department of Medical Science, Policlinico di Monserrato, University of Cagliari; D.D. Gladman, MD, FRCPC, Professor of Medicine, University of Toronto, and Senior Scientist, Krembil Research Institute, and Director, Psoriatic Arthritis Program University Health Network; A. Mathieu, MD, Professor of Rheumatology and Head of Rheumatology Unit, University of Cagliari; G. Porru, MD, Department of Medical Science, Policlinico di Monserrato, University of Cagliari; P.P. Tak, MD, PhD, Professor of Rheumatology, Academic Medical Center/University of Amsterdam; C. Sardu, MD, Department of Public Health, University of Cagliari; R. Scarpa, MD, Professor of Rheumatology, Rheumatology Research Unit, Department of Clinical and Experimental Medicine, University "Federico II"; A. Marchesoni, MD, Department of Rheumatology, ASST Gaetano Pini-CTO; W.J. Taylor, PhD $M B C h B, F R A C P, F A F R M$, Rehabilitation Teaching and Research Unit, and Department of Medicine, Wellington School of Medicine and Health Sciences, University of Otago; C. Salvarani, MD, Professor of Rheumatology, Rheumatology Unit, Azienda USL-IRCCS di Reggio Emilia and Università di Modena e Reggio Emilia; J. Kalden, MD, Medizinische Klinik III mit Poliklinik, Friedrich-Alexander-Universität ErlangenNuernberg; E. Lubrano, MD, PhD, Associate Professor of Rheumatology, Dipartimento di Medicina e Scienze della Salute, Università degli Studi del Molise; S. Carneiro, MD, PhD, Dermatologist and Rheumatologist, HUCFF Federal University of Rio de Janeiro, and Associate Professor of

Personal non-commercial use only. The Journal of Rheumatology Copyright ()$_{2018}$. All rights reserved. 
Psoriatic arthritis (PsA) is a frequent and potentially disabling chronic inflammatory disease affecting the musculoskeletal (MSK) system (peripheral joints, digits, spine, entheses), skin, and nails ${ }^{1,2}$.

In the last decade, disease assessment research has yielded improved stratification of PsA manifestations into discrete clinical domains, to more comprehensively assessed disease activity with reliable outcome measures for randomized controlled trials (RCT) and longitudinal observational studies (LOS) as well as routine management ${ }^{3}$. They include peripheral arthritis, enthesitis, dactylitis, axial disease, skin and nails, as well as a variety of patient-reported domains such as fatigue, function, and quality of life. Efficacy of approved and emerging medications for the treatment of PsA clinical domains, using these outcome measures, has been demonstrated in the Group for Research and Assessment of Psoriasis and Psoriatic Arthritis (GRAPPA) treatment recommendations ${ }^{4}$.

GRAPPA consists of members from different professional backgrounds, including rheumatologists, dermatologists, and other investigators ${ }^{5}$, and includes among its aims the validation and standardization of outcome assessment tools in PsA and psoriasis. In 2007, GRAPPA and OMERACT (Outcome Measures in Rheumatoid Arthritis Clinical Trials), after a multistep process ${ }^{6,7,8}$, reached consensus on a core set of 6 domains to be measured in all clinical trials. These included peripheral joint activity, skin activity, pain, patient's global assessment (PtGA), physical function, and health-related quality of life (HRQOL). Several other domains were considered important, not mandatory, but preferably were assessed at some point in a clinical trial development program: spinal disease, dactylitis, enthesitis,

Dermatology, School of Medical Sciences, State University of Rio de Janeiro; M. Piga, MD, Department of Medical Science, Policlinico di Monserrato, University of Cagliari; A. Floris, MD, Rheumatology Unit, University of Cagliari; F. Desiati, MD, Department of Rheumatology, ASST Gaetano Pini-CTO; J.A. Flynn, MD, MBA, Med, Professor of Medicine, Director Spondyloarthritis Program, Johns Hopkins University, School of Medicine; S. D'Angelo, MD, Rheumatology Department of Lucania, San Carlo Hospital of Potenza and Madonna delle Grazie Hospital of Matera Italy, and PhD Scholarship in Health Sciences, Department of Health Sciences, University of Molise; A.W. van Kuijk, MD, PhD, Amsterdam Rheumatology and Immunology Center Reade; M.G. Catanoso, MD, Rheumatology Unit, Azienda USL-IRCCS di Reggio Emilia and Università di Modena e Reggio Emilia; F. Caso, MD, PhD, Assistant Professor, Rheumatology Research Unit, Department of Clinical and Experimental Medicine, University "Federico II"; P. Contu, MD, Department of Public Health, University of Cagliari; I. Ujfalussy, MD, Department of Rheumatology Semmelweis University; P.S. Helliwell, MD, PhD, Leeds Institute of Molecular Medicine, University of Leeds;

P.J. Mease, MD, Swedish Medical Center Rheumatology Research Division; Clinical Professor, University of Washington School of Medicine. Ignazio Olivieri, MD, died July 28, 2017; he was Director of the Rheumatology Department of Lucania, San Carlo Hospital of Potenza and Madonna delle Grazie Hospital of Matera.

Address correspondence to Prof. A. Cauli, Dipartimento di Scienze

Mediche, Università di Cagliari, ss 554, Monserrato 09042, Italy.

E-mail:cauli@medicina.unica.it

Accepted for publication February 28, 2018. fatigue, nail disease, radiography, acute-phase reactants, and physician's global assessment (PGA). Recently, OMERACT members voted that the PsA core set should be revised because of updated knowledge about PsA, but in particular because of the strong feeling that patient input needed to be incorporated more strongly ${ }^{9}$. The new final core set included 3 parts: (1) an inner group of domains to be measured in all RCT and LOS, including MSK disease activity (peripheral arthritis, dactylitis, enthesitis, and axial symptoms), skin disease activity (skin psoriasis and nail dystrophy), pain, PtGA, physical function, HRQOL, fatigue, and systemic inflammation; (2) a middle circle of domains, important but not mandatory, including economic costs, emotional well-being, participation, and structural damage; and (3) an outer circle represents the research agenda (stiffness, independence, treatment burden, and sleep; Table 1) ${ }^{10}$.

The PGA has been removed from the revised Core Domain Set and a preponderance of objective and patient-reported outcomes measurements (PRO) is now evident. PGA of disease summarizes physician clinical assessment and incorporates patient feedback to the doctor and is therefore a major determinant that can influence therapy choice and general management. The deletion of

Table 1. 2006 versus 2016 GRAPPA-OMERACT PsA Core Domain Sets.

\begin{tabular}{lc}
\hline Inner Circle (2006) & 2016 \\
\hline Peripheral joint activity & MSK disease activity \\
Skin activity & Skin disease activity \\
Pain & Pain \\
Patient's global & Patient's global \\
Physical function & Physical function \\
HRQOL & HRQOL \\
& Fatigue \\
\hline Middle Circle (2006) & 2016 \\
\hline Enthesitis & Participation \\
Dactylitis & Emotional well-being \\
Spine & Economic costs \\
Nails & Structural damage \\
Physician's global & \\
Fatigue & \\
Radiology & \\
Acute-phase reactants & \\
\hline Outer Circle (2006) & Stiffness \\
\hline Participation & Tndependence \\
Tissue analysis & Sleep burden \\
MRI & \\
Computed tomography & \\
Ultrasound & \\
\hline
\end{tabular}

GRAPPA: Group for Research and Assessment of Psoriasis and Psoriatic Arthritis; OMERACT: Outcome Measures in Rheumatology Clinical Trials; PsA: psoriatic arthritis; MSK: musculoskeletal; HRQOL: health-related quality of life; MRI: magnetic resonance imaging. 
PGA as a domain item is therefore of note, given that physician judgment in the recommendation of and taking responsibility for treatment is important. Notably, previous studies have shown a discordance between patient and physician assessment in arthritis, raising important questions of the consequences in treatment decisions ${ }^{11}$.

Following GRAPPA input, we previously conducted a study that demonstrated that PtGA by means of $0-100 \mathrm{~mm}$ visual analog scale (VAS), taking into account both MSK and skin disease, was a reliable instrument to assess the burden of PsA disease ${ }^{12}$. Given the relative independence of skin and MSK manifestations, it was proposed that in PsA, it is appropriate to assess the effect of dermatologic and MSK involvement segregated into 2 separate questions, 1 addressing MSK and 1 addressing the skin. We further analyzed the data collected during the GRAPPA PtGA study following the same methodology and focusing on the global evaluation of PsA disease activity from the physician's point of view to validate the instrument, and finally to compare the physician perspective with the patient perspective by means of these 2 instruments.

\section{MATERIALS AND METHODS}

Patients. Patient selection and methodology was previously described in detail $^{12}$. Briefly, 319 consecutive patients $(58 \%$ male, $42 \%$ female; mean \pm SD age $51 \pm 13 \mathrm{yrs}$ ) fulfilling the ClASsification for Psoriatic ARthritis criteria ${ }^{2}$ were enrolled in 17 centers from 8 countries worldwide (Italy, the United States, Canada, the Netherlands, New Zealand, Germany, Brazil, and the United Kingdom). Seventeen centers were rheumatology units; only 1 center was a dermatology unit, but the local investigator is also a rheumatologist and performed the assessment of the enrolled patients. Consecutive patients with PsA were included in the study, regardless of disease activity, treatment, and clinical subsets, as defined according to Moll and Wright ${ }^{1,12}$.

Questionnaires. Physician perception of disease was investigated following specific questions by means of 0-100 mm VAS as a global score (PGA), encompassing joints (regarded as all MSK manifestations, therefore including peripheral joints, dactylitis, enthesitis, and axial, to keep it simple, as suggested by the patients) and all aspects of skin disease (including nails), as well as a question specific to joint manifestations and skin (PhysMSK and PhysSk, respectively; Table 2). The questionnaires were elaborated by "expert opinion" consensus among GRAPPA members. In non-English speaking countries, the coordinator of the center was responsible for the process of translation/back translation of the questionnaires. The questionnaires, related to the degree of disease activity, were administered at baseline and after 1 week, without any change in treatment, to test the reliability of the instrument. The 1-week interval was selected as a good compromise to avoid the repetition of the previous score by the physician and modification of disease activity. The 3 different VAS questionnaires were administered on the same day of the clinical examination, in a changing random order to exclude any bias.

Clinical assessment. Detailed clinical evaluation was performed according to a specific protocol. Demographic data and medical history were taken at baseline. Joint disease clinical subsets were defined according to Moll and Wright ${ }^{1}$. MSK manifestations were objectively evaluated by means of the American College of Rheumatology joint count (68 tender, 66 swollen) for peripheral joint. Dactylitis and enthesitis were also clinically assessed (presence or absence) and detailed in the clinical record form. The Bath Ankylosing Spondylitis Disease Activity Index was used in patients with axial involvement because this instrument has been shown to be reliable in
Table 2. PGA, PhysMSK, and PhysSk questionnaires.

Physician VAS

- Global How would you rate global skin and MSK disease in this patient? (10-cm line)

Quiet __ Very severe

- MSK How would you rate MSK disease in this PsA patient? (10-cm line)

Quiet___ Very severe

- Skin How would you rate skin disease in this PsA patient? (10-cm line)

Quiet__ Very severe

PGA: physician's global assessment; PhysMSK: physician's musculoskeletal assessment; PhysSk: physician's skin assessment; VAS: visual analog scale; MSK: musculoskeletal; PsA: psoriatic arthritis.

$\mathrm{PsA}^{13}$. Skin psoriasis was evaluated by means of the Psoriasis Area and Severity Index $(\mathrm{PASI})^{14}$. Involvement of the face, genitalia, hands, buttocks/intergluteal, or foot was also recorded. Drug treatment at time of enrollment was detailed.

Statistical analysis. Descriptive analysis was performed, expressing variables as mean $\pm \mathrm{SD}$, or median with 25 th and 75 th percentiles, according to data distribution. Intraclass correlation coefficient (ICC) was used to assess the concordance between VAS score at Week 0 and at Week 1. Pearson correlation analysis was carried out to evaluate the strength of association between PhysMSK and PhysSk as well as PGA versus PtGA, PhysMSK versus patient (PMSK), and PhysSk versus patient skin (PSk).

The influence of PhysMSK and PhysSk on PGA was analyzed through a multiple linear regression. PhysMSK and PhysSk were considered independent variables, while PGA was the dependent variable. A multiple linear regression was also carried out to estimate the influence of these factors on on PhysMSK and/or PhysSk: sex, age, job (manual, intellectual, contact with public), dactylitis (presence vs absence), enthesitis (presence vs absence), number of tender joints, number of swollen joints, arthritis duration, PASI score, areas of body surface involved (face, genitalia, hands, buttocks and/or intergluteal, feet), and psoriasis duration.

Kruskall-Wallis test was performed to analyze differences in values of a continuous variable (PhysMSK, PhysSk) between clinical subsets.

Ethics. Ethical committee approval was obtained from the following centers: Johns Hopkins University, Office of Human Subjects Research, Institutional Review Boards, Study \#: NA_00026662; Toronto University Health Network Research Ethics Board \#08-0630-AE; Medical Ethical Committee of the Academic Medical Centre/University of Amsterdam (ref: MEC 05/162, ISRCTN23328456); and New Zealand Central Region Ethics Committee (approval number CEN/06/03/016). In the other participating centers, Ethical Committee Approval was not required in accordance with the policy of the institutions and local legislation for this kind of study.

\section{RESULTS}

Seventeen centers from 10 countries participated in this study. A total of 319 patients with PsA gave informed consent and were included in the analysis. For MSK involvement, the median number of tender joints was $5(1-13)$, median number of swollen joints was $1(0-5)$, axial involvement was reported in $8 \%$, dactylitis was present in $7 \%$, and enthesitis in $21 \%$ of patients. For skin involvement, the median PASI score was $2.8(0.7-6.5)$ in overall cohort and $4.0(2.0-5.2)$ in the single dermatology unit $(\mathrm{p}=$ not significant $)$. Face, hands, buttocks/intergluteal, genitalia, and feet involvement was reported in $13 \%, 24 \%, 18 \%, 5 \%$, and $19 \%$ of the patients, respectively. Patient treatment was as follows: $63 \%$ were 
receiving conventional synthetic disease-modifying antirheumatic drugs (DMARD), 23\% biological DMARD, 37\% nonsteroidal antiinflammatory drugs, and 9\% steroids. Detailed clinical data of the patients have been described previously ${ }^{12}$. Median baseline values for the 3 questionnaires were as follow: PGA 32 (19-50), PhysMSK 25 (11-47), PhysSk 20 (9-40).

Test-retest. The ICC coefficients revealed good reproducibility of the VAS measures. ICC for PGA was equal to 0.90 (95\% CI $0.86-0.92$ ), ICC for PhysMSK was equal to 0.86 (95\% CI 0.81-0.89), and ICC for PhysSk was equal to 0.67 (95\% CI 0.58-0.75).

Physician's global assessment. To quantify the specific influence of the MSK and skin component in the PGA of disease, we performed a multiple linear regression analysis. The final regression model was statistically significant, $\mathrm{p}<0.0001$ and $\mathrm{R}^{2}=0.78$. The analysis showed $\mathrm{B}$ coefficient 0.69 (95\% CI 0.64-0.74) for PhysMSK and B coefficient 0.32 (95\% CI 0.27-0.37) for PhysSk, meaning that the MSK component is perceived as the dominant clinical issue compared to psoriasis.

Physician assessment of joint disease. To test the specific influence on PhysMSK of swollen and tender joints, dactylitis, enthesitis, arthritis duration, patient sex, age, and job, a multiple linear regression was performed. Enthesitis, arthritis duration, sex, age, and job were not significantly associated with PhysMSK, and therefore were eliminated from the model. The final regression model $(p<0.0001)$ included swollen joints, tender joints, and dactylitis. The $\mathrm{R}^{2}$ value was 0.34 ; the regression coefficients were B $1.66(95 \%$ CI 1.19-2.12) for swollen joints, B 0.27 (95\% CI 0.05-0.49) for tender joints, and B 9.60 for dactylitis (95\% CI 2.64-16.56).

Further, patients were also grouped according to number of joints involved: 1-3 joints (median PhysMSK 10 and 15.5 for tender and swollen, respectively); 4-5 joints (median PhysMSK 20.5 and 35.5 for tender and swollen, respectively); > 5 joints (median PhysMSK 39 and 52.5 for tender and swollen, respectively), showing higher VAS scores for patients with more joints involved $(\mathrm{p}<0.0001)$. Involvement of metacarpal phalangeal joints, wrist, and proximal interphalangeal joints of hands and feet were perceived as associated with more severe disease.

The detailed median PhysMSK in the different clinical subsets were as follow: polyarticular 27 (15-46), oligoarticular 20 (7-40), axial 40 (21-55), distal 30 (20-50), mutilans 44 (8-71), and more than 1 subset 25 (11-41), with no statistically significant differences.

Physician assessment of skin disease. To test the specific influence on skin physician assessment of PASI score; involvement of face, genitalia, hands, buttocks and/or intergluteal, feet; psoriasis duration; patient sex; age; and job; a multiple linear regression was performed. These were not significantly associated with PhysSk, and therefore were eliminated from the model: hands and genitalia skin involvement, psoriasis duration, sex, age, and job. The final regression model $(\mathrm{p}<0.0001)$ included the 4 independent variables PASI score, face, buttocks or intergluteal, and foot involvement $\left(\mathrm{R}^{2}\right.$ value $\left.=0.61\right)$. The regression coefficients were B 2.47 (95\% CI 2.19-2.76) for PASI, B 6.62 (95\% CI 1.34-11.91) for face involvement, B 6.93 (95\% CI 2.32-11.55) for buttocks or intergluteal involvement, and B 7.92 (95\% CI 3.18-12.67) for foot involvement.

The detailed median PhysSk in the different clinical subsets were as follow: polyarticular 17 (9-40), oligoarticular 12 (4-31), axial 30 (13-55), distal 20 (10-53), mutilans 36 (8-55), and more than 1 subset 25 (11-40), with no statistically significant differences.

MSK versus skin psoriasis. To investigate physician clinical perception of MSK disease compared to skin disease, we correlated PhysMSK and PhysSk assessment. The analysis revealed that, according to clinical judgment, MSK and skin disease do not correlate regarding disease activity as evidenced by the correlation coefficient value between PhysMSK and PhysSk, which resulted in $r=0.24$, with a marked scattered pattern at the dot plot (Figure 1).

Physician assessment compared with patient self-assessment. To test the possible agreement or disagreement in global, MSK, or skin evaluation by physician compared to patient self-assessment, we correlated PGA versus PtGA $(r=0.36)$, PhysMSK versus PMSK $(r=0.39)$, and PhysSk versus PSk $(r=0.49)$, by means of Pearson's test (the ideal concordance would be $r=1$ ). A marked scattered pattern of the dot blots (mainly in the lower/right part of the graph, indicating higher values scored by the patients) is clearly visible, indicating a poor correlation between the physician and patient evaluations (Figure 2).

\section{DISCUSSION}

The assessment of PsA poses a challenge to the clinician because of its varied manifestations including peripheral joints, axial disease, enthesitis, dactylitis, and dermatological ones.

A trend has been clearly observed to include more PRO in general and even domain-specific assessment of diseases, such as joint count in rheumatoid arthritis (RA) ${ }^{15}$, although the data available in the literature are debatable ${ }^{11,16}$. Chaudhry, et $a l^{17}$ conducted a study comparing the degree of agreement between patient and physician in evaluating joint disease activity and damage as well as the degree of skin disease. They concluded that PsA patient self-report evaluation has a poor correlation with physician assessment, and therefore expert physical examination should remain the gold standard for the assessment of actively inflamed joint and skin disease. Several other studies have been performed to determine whether patient evaluation, given the emotional component and the difficulties in differentiating between

Personal non-commercial use only. The Journal of Rheumatology Copyright @ 2018 . All rights reserved. 


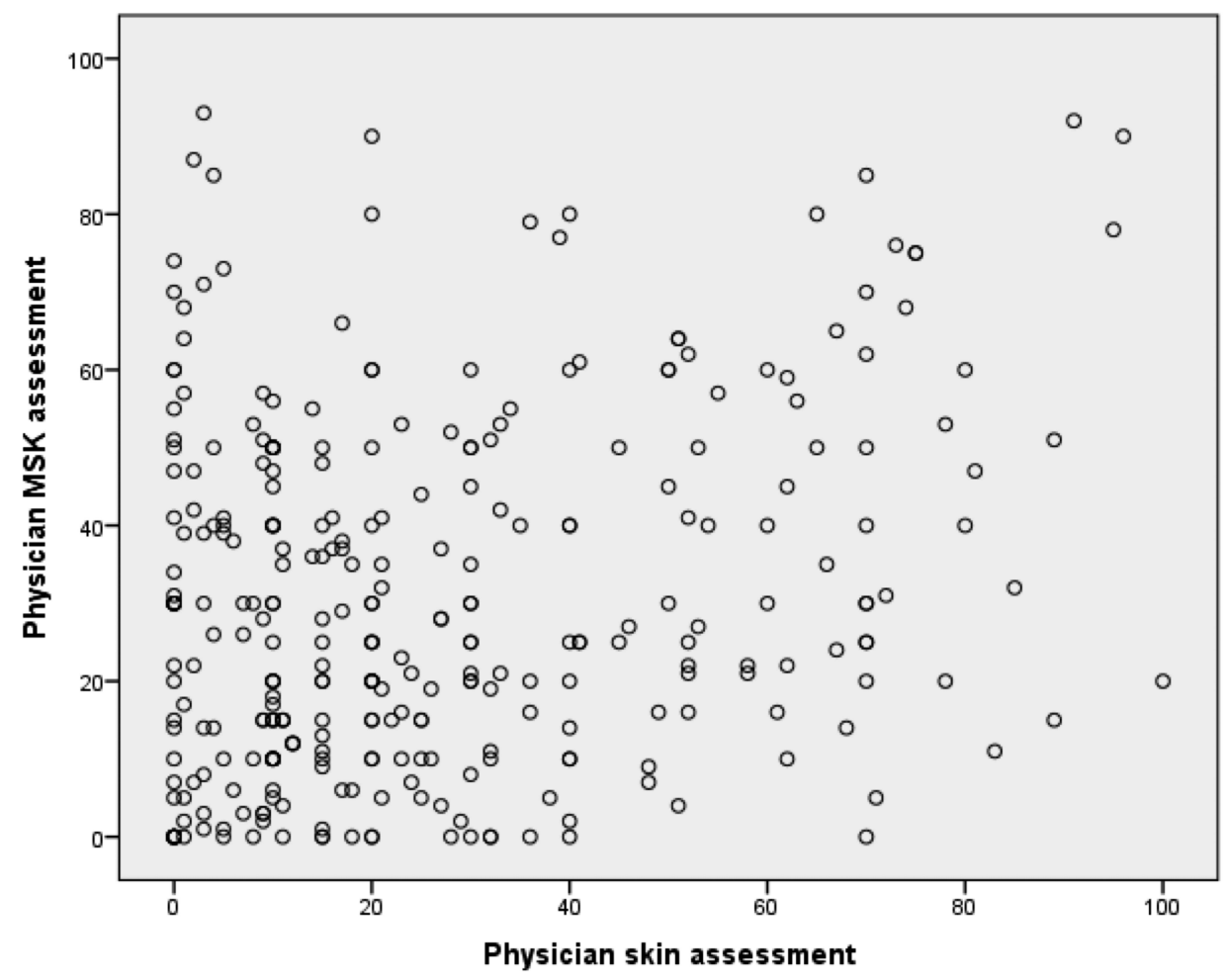

Figure 1. Physician perception of MSK disease versus physician perception of skin disease $\left(\mathrm{R}^{2}=0.24\right)$. A scattered pattern is clearly visible, indicating lack of correlation of MSK and skin disease. MSK: musculoskeletal.

possible multiple sources of symptoms, is accurate and in line with physician evaluation. Furst, et al found that among 305 paired patient-physician records analyzed, 23\% were misaligned mainly because of a higher perception of disease activity and disability by the patients ${ }^{18}$, while Eder, et al reported that fatigue, pain, disability, and joint counts were the most important factors responsible for discrepancy between patient and physician joint assessment ${ }^{19}$. On the other hand, Dandorfer, et al found good correlations of patient's and physician's assessment of disease when using VAS scores, although physicians usually evaluated the disease activity of PsA lower than the patients ${ }^{20}$, as reported also by Desthieux, et $a l^{21}$.

Physician perception of disease activity, following history-taking and objective examination of the patient including laboratory and imaging evaluation, is partly independent of patient self-assessment, and thus should be factored into the determination of clinical management and treatment. PGA and PtGA are both reliable instruments for assessing disease activity, but they do not overlap, reflecting the 2 slightly different sides of the same coin.

The 100-mm VAS is recommended over a 5-point Likert and 11-point numeric rating scale as the instrument for the PtGA and PGA domain because of demonstrated psychometric quality in RA and osteoarthritis ${ }^{22,23}$. Reliability was determined by test-retest.
Our present study demonstrated that PGA assessed by means of VAS is a reliable tool related to both MSK and dermatological disease activity. Because MSK and skin disease very often diverge, it is suggested that both PhysMSK and PhysSk are also evaluated separately for a more detailed analysis of the physician perspective.

The specific influence of the joint and skin components in the physician's global perception of disease was evaluated by multiple linear regression analysis. It should be emphasized that PhysMSK and PhysSk explain nearly all the variance in PGA $\left(R^{2}=0.78\right)$. It also showed a preponderance of the arthritis symptoms over the skin, not surprising given the low PASI scores found in the majority of the patients attending rheumatology clinics, and in the PsA population in general. It is noteworthy that 17 out of 18 centers of the consortium were Rheumatology Units rather than Dermatology Units; this could represent a bias in the recruitment process, which may explain a more severe joint disease. On the other hand, many patients recruited by the rheumatologists were also followed by a dermatologist, and it is unlikely that patients with arthritis were followed only by a dermatologist. For these reasons, we believe that our cohort of patients well represents the general population of patients with PsA.

Further analysis of the assessment of joint disease by the physician showed that statistically significantly higher values

Personal non-commercial use only. The Journal of Rheumatology Copyright ()$_{2018}$. All rights reserved. 
a.

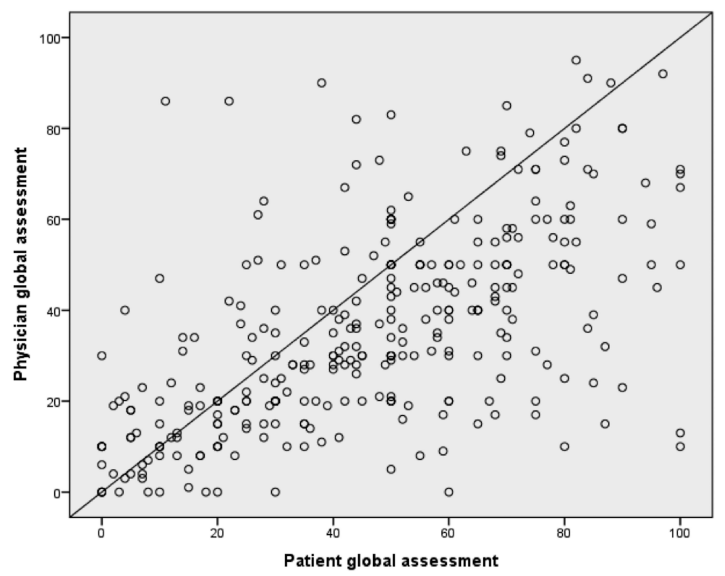

b.

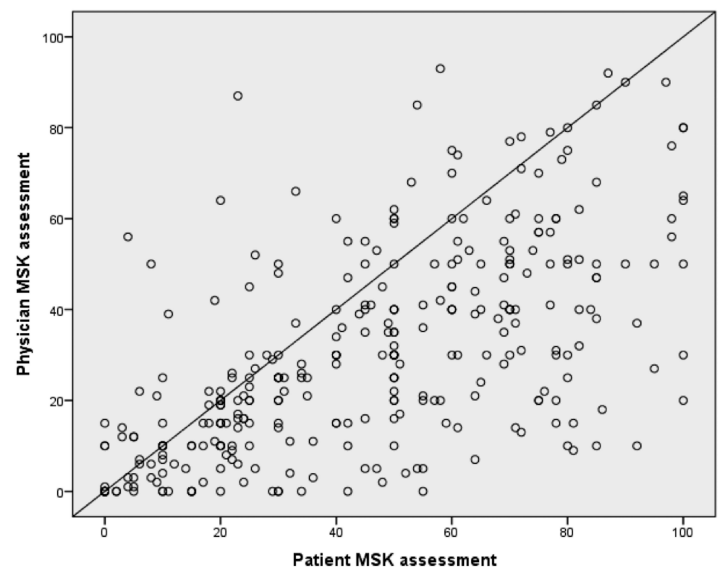

c.

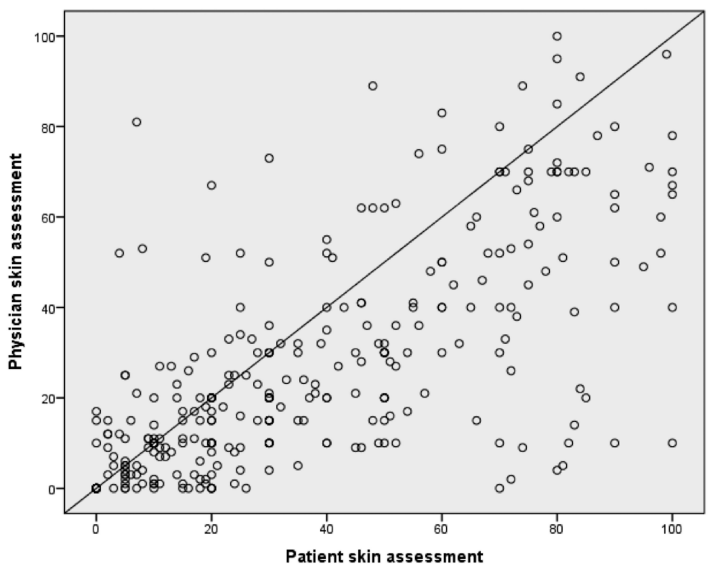

Figure 2. Physician's versus patient's perception of global (A), MSK (B), and skin disease (C). A scattered pattern, mainly in the lower/right part of the graphs, indicates poor correlation between physicians and patients (who scored higher). MSK: musculoskeletal

of VAS scores correlated with the number of joints involved, supporting the validity of the instrument (VAS) for the domain of interest (physician joint assessment). Further, there was no relationship to a particular subset of clinical type of PsA. Indeed, patients belonging to the mutilans subset had higher scores followed by patients of the axial subset, but without statistical significance.

It is noteworthy that the occurrence of dactylitis, in our cohort, was perceived by the physician as severe. Dactylitis is a typical clinical feature of PsA (being present in 16-48\% of cases) and can be considered a clinical indicator of disease severity ${ }^{24}$. On the other hand, the presence of enthesitis, arthritis duration, sex, age, and job of the patient did not influence physician perception of joint disease.

A similar approach was followed to analyze physician assessment of the effect of skin disease in relation to the degree of skin psoriasis by means of PASI, involvement of face, genitalia, hands, buttocks and/or intergluteal, feet, psoriasis duration, sex, age, and job. A significant association was found for PASI score and involvement of the face, buttocks or intergluteal, and feet, showing $\mathrm{R}^{2}$ value 0.57 , indicating that these variables accounted for $57 \%$ of the total variation in PhysSk. PASI scores do not differentiate based on the involved area, but the physician perception of disease severity clearly is also dependent on the involvement of precise areas, not simply on the "amount" of involved skin. In our study, the sites of major effect on physician perception were the face, buttocks, intergluteal, and feet, probably because of their involvement in working and life activities, hygiene, and also in social interaction. It should be underlined that these findings were derived from a cohort characterized by a low PASI score, as is typical in patients with PsA.

We further investigated the effect of arthritis compared to skin psoriasis. The analysis revealed that joint and skin disease did not correlate in terms of disease activity, a finding consistent with other studies ${ }^{25}$. Some synthetic DMARD work better for 1 manifestation but not the other, such as cyclosporine for the skin and leflunomide for the joints ${ }^{26,27}$, and the same is true for some biologics ${ }^{4}$. Genetic factors may also differ, as well as immune cells implicated in disease pathogenesis ${ }^{28,29}$. Clinical and experimental evidence suggests that different mechanisms drive the joint and skin processes, but the lack of knowledge of the causative agent(s) does not allow testing of this hypothesis. The lack of correlation between the joint and skin disease scores (objective, but also perceived by the treating physician) raises the point that although the PGA performed well overall in our study as a single measure, a more complete assessment may be derived from also measuring PhysMSK and PhysSk, for example in the circumstance that a drug may improve one of these domains but not adequately the other.

Finally, our study has underlined the frequent discordance between physician and patient in the evaluation of PsA. Patients are prone to score a higher burden of disease compared to physician evaluation. The reasons that may explain this discrepancy are several: physicians may have seen worse cases or may be used to treat other more life-threatening disease, while patients do consider their

Personal non-commercial use only. The Journal of Rheumatology Copyright (C) 2018. All rights reserved. 
everyday disease and difficulties as reference. This mismatch has critical consequences because it is the physician who proposes the treatment approach to the patient, and takes the responsibility for the prescription.

PGA, as well as PhysMSK and PhysSk separately, are reliable in PsA. Although the PGA as a single measure was demonstrated to perform well in assessing the patient in totality, it was also demonstrated that physician assessment of joint and skin disease activity may be divergent. Therefore, although PGA is an acceptable single measure for clinical trials and clinical practice, in situations such as the study or common use of a drug that may improve the joints but not the skin, it would be important to assess both the PhysMSK and PhysSk as well.

\section{REFERENCES}

1. Wright V, Moll JM. Psoriatic arthritis. In: Wright V, Moll JM, eds. Seronegative polyarthritis. Amsterdam: North Holland Publishing Co.; 1976:169-223.

2. Taylor W, Gladman D, Helliwell P, Marchesoni A, Mease P, Mielants H; CASPAR Study Group. Classification criteria for psoriatic arthritis. Arthritis Rheum 2006;54:2665-73.

3. Gladman DD, Mease PJ, Healy P, Helliwell PS, Fitzgerald O, Cauli A, et al. Outcome measures in psoriatic arthritis. J Rheumatol 2007;34:1159-66.

4. Coates LC, Kavanaugh A, Mease PJ, Soriano ER, Laura Acosta-Felquer M, Armstrong AW, et al. Group for Research and Assessment of Psoriasis and Psoriatic Arthritis 2015 treatment recommendations for psoriatic arthritis. Arthritis Rheumatol 2016;68:1060-71.

5. Jadon DR, Gladman DD, Mease PJ, Fitzgerald O, Chandran V, Goel $\mathrm{N}$, et al. Proceedings of the GRAPPA 2016 retreat. J Rheumatol 2017;44:668-73.

6. Taylor WJ. Preliminary identification of core domains for outcome studies in psoriatic arthritis using Delphi methods. Ann Rheum Dis 2005;64 Suppl 2:ii110-2.

7. Gladman DD, Mease PJ, Krueger G, van der Heidje DM, Antoni C, Helliwell PS, et al. Outcome measures in psoriatic arthritis. J Rheumatol 2005;32:2262-9.

8. Gladman DD, Mease PJ, Strand V, Healy P, Helliwell PS, Fitzgerald $\mathrm{O}$, et al. Consensus on a core set of domains for psoriatic arthritis. J Rheumatol 2007;34:1167-70

9. Orbai AM, de Wit M, Mease PJ, Callis Duffin K, Elmamoun M, Tillett W, et al. Updating the psoriatic arthritis (PsA) core domain set: a report from the PsA workshop at OMERACT 2016. J Rheumatol 2017;44:1522-8.

10. Ogdie A, de Wit M, Callis Duffin K, Campbell W, Chau J, Coates LC, et al. Defining outcome measures for psoriatic arthritis: a report from the GRAPPA-OMERACT working group. J Rheumatol 2017;44:697-700.

11. Hewlett SA. Patients and clinicians have different perspectives on outcomes in arthritis. J Rheumatol 2003;30:877-9.

12. Cauli A, Gladman DD, Mathieu A, Olivieri I, Porru G, Tak PP, et al; GRAPPA 3PPsA Study Group. Patient global assessment in psoriatic arthritis: A multicenter GRAPPA and OMERACT study. J Rheumatol 2011;38:898-903.
13. Gladman DD, Cook RJ, Schentag C, Feletar M, Inman RI, Hitchon $\mathrm{C}$, et al. The clinical assessment of patients with psoriatic arthritis: results of a reliability study of the spondyloarthritis research consortium of Canada. J Rheumatol 2004;31:1126-31.

14. Fredriksson T, Pettersson U. Severe psoriasis - oral therapy with a new retinoid. Dermatologica 1978;157:238-44.

15. Pincus T, Yazici Y, Bergman MJ. RAPID3, an index to assess and monitor patients with rheumatoid arthritis, without formal joint counts: similar results to DAS28 and CDAI in clinical trials and clinical care. Rheum Dis Clin North Am 2009;35:773-8.

16. Bossert M, Prati C, Vidal C, Bongain S, Toussirot E, Wendling D. Evaluation of self-report questionnaires for assessing rheumatoid arthritis activity: a cross-sectional study of RAPID3 and RADAI5 and flare detection in 200 patients. Joint Bone Spine 2012;79:57-62.

17. Chaudhry SR, Thavaneswaran A, Chandran V, Gladman DD. Physician scores vs patient self-report of joint and skin manifestations in psoriatic arthritis. Rheumatology 2013;52:705-11.

18. Furst DE, Tran M, Sullivan E, Pike J, Piercy J, Herrera V, et al. Misalignment between physicians and patient satisfaction with psoriatic arthritis disease control. Clin Rheumatol 2017;36:2045-54.

19. Eder L, Thavaneswaran A, Chandran V, Cook R, Gladman DD. Factors explaining the discrepancy between physician and patient global assessment of joint and skin disease activity in psoriatic arthritis patients. Arthritis Care Res 2015;67:264-72.

20. Dandorfer SW, Rech J, Manger B, Schett G, Englbrecht M. Differences in the patient's and the physician's perspective of disease in psoriatic arthritis. Semin Arthritis Rheum 2012;42:32-41.

21. Desthieux C, Granger B, Balanescu AR, Balint P, Braun J, Canete $\mathrm{JD}$, et al. Determinants of patient-physician discordance in global assessment in psoriatic arthritis: a multicenter European study. Arthritis Care Res 2017;69:1606-11.

22. Bellamy N, Campbell J, Syrotuik J. Comparative study of self-rating pain scales in rheumatoid arthritis patients. Curr Med Res Opin 1999;15:121-7.

23. Bellamy N, Campbell J, Syrotuik J. Comparative study of self-rating pain scales in osteoarthritis patients. Curr Med Res Opin 1999;15:113-9.

24. Brockbank JE, Stein M, Schentag CT, Gladman DD. Dactylitis in psoriatic arthritis: a marker for disease severity. Ann Rheum Dis 2005;64:188-90.

25. Oriente P, Biondi-Oriente C, Scarpa R. Psoriatic arthritis. Clinical manifestations. Baillieres Clin Rheumatol 1994;8:277-94.

26. Spadaro A, Riccieri V, Sili-Scavalli A, Sensi F, Taccari E, Zoppini A. Comparison of cyclosporin A and methotrexate in the treatment of psoriatic arthritis: a one-year prospective study. Clin Exp Rheumatol 1995;13:589-93.

27. Kaltwasser JP, Nash P, Gladman D, Rosen CF, Behrens F, Jones P, et al; Treatment of Psoriatic Arthritis Study Group. Efficacy and safety of leflunomide in the treatment of psoriatic arthritis and psoriasis: a multinational, double-blind, randomized, placebo-controlled clinical trial. Arthritis Rheum 2004;50:1939-50.

28. Pitzalis C, Cauli A, Pipitone N, Smith C, Barker J, Marchesoni A, et al. Cutaneous lymphocyte antigen-positive T lymphocytes preferentially migrate to the skin but not to the joint in psoriatic arthritis. Arthritis Rheum 1996;39:137-45.

29. Cauli A, Pitzalis C, Yanni G, Awad M, Panayi GS. CD1 expression in psoriatic and rheumatoid arthritis. Rheumatology 2000; 39:666-73. 